\title{
ESTRUTURA DO COMPONENTE ARBUSTIVO-ARBÓREO DE DOIS ESTÁDIOS SUCESSIONAIS DE FLORESTA ESTACIONAL SEMIDECIDUAL NA RESERVA FLORESTAL MATA DO PARAÍSO, VIÇOSA, MG, BRASIL ${ }^{1}$
}

\author{
Sheila Isabel do Carmo Pinto ${ }^{2}$, Sebastião Venâncio Martins ${ }^{3}$, Aderbal Gomes da Silva ${ }^{4}$, Nairam Felix de \\ Barros $^{5}$, Herly Carlos Teixeira Dias ${ }^{3}$ e Leandro Moraes Scoss ${ }^{6}$
}

\begin{abstract}
RESUMO - Realizou-se o estudo das variações estruturais do componente arbustivo- arbóreo em dois estádios sucessionais - inicial e madura - de Floresta Estacional Semidecidual, na Reserva Florestal Mata do Paraíso, em Viçosa, MG, Brasil. A Reserva Florestal está situada nas coordenadas $20^{\circ} 45^{\prime} \mathrm{S}$ e $42^{\circ} 55^{\prime} \mathrm{W}$ e a uma altitude média de $689 \mathrm{~m}$. O clima da região é classificado como Cwb pelo sistema de Köppen. As espécies arbustivoarbóreas foram amostradas dentro de 20 parcelas de 10 × $30 \mathrm{~m}$, sendo 10 parcelas em cada estádio sucessional, sendo considerados apenas os indivíduos com diâmetro a $1,30 \mathrm{~m}$ do solo (DAP) $\geq 4,8 \mathrm{~cm}$. Na floresta inicial foram amostrados 399 indivíduos, distribuídos em 27 famílias e 55 espécies. As espécies com maior valor de importância (VI) foram Piptadenia gonoacantha, Vernonanthura diffusa, Miconia cinnamomifolia, Piptocarpha macropoda e Luehea grandiflora. O índice de diversidade de Shannon (H') foi de 3,31 nat.ind. ${ }^{-1}$ e a equabilidade de Pielou (J'), igual a 0,83. No estádio floresta madura foram amostrados 623 indivíduos, distribuídos em 31 famílias e 78 espécies. As espécies com maior valor de importância (VI) foram Euterpe edulis, Piptadenia gonoacantha, Nectandra lanceolata, Myrcia sphaerocarpa e Guapira opposita. O índice de diversidade de Shannon (H') foi de 3,46 nat.ind. ${ }^{-1}$ e a equabilidade de Pielou (J'), igual a 0,79. As distribuições diamétricas das quatro espécies mais abundantes em cada estádio sucessional apresentaram padrões distintos, aparentemente relacionados ao estádio sucessional.
\end{abstract}

Palavras-chave: Floresta Estacional Semidecidual, estrutura comunitária e fitossociologia.

\section{STRUCTURE OF THE TREE-SHRUB COMPONENT IN TWO SUCCESSIONAL STAGES OF SEMIDECIDUOUS FOREST IN THE MATA DO PARAÍSO FOREST RESERVE, VICOSA, MG, BRAZIL}

\begin{abstract}
The study of the floristic and structural variations of the tree-shrub component was carried out in two successional stages of Semideciduous Forest, initial and mature, located in the Mata do Paraíso Forest Reserve, in Viçosa, Minas Gerais State, Brazil. The Forest Reserve is located at $20^{\circ} 45^{\prime}$ 'S and $42^{\circ} 55^{\prime} \mathrm{W}$, with average altitude of $689 \mathrm{~m}$. The regional climate is classified as Cwb by the Köppen system. Individual trees with $\geq 4.8 \mathrm{~cm}$ diameter at breast height $(\mathrm{DBH})$ were sampled in $20-10 \times 30 \mathrm{~m}$ plots (10 plots in each site). In the initial forest, 399 individuals were identified with distribution among 27 families and 55 species. Based on the importance value (IV), the most representative species were Piptadenia gonoacantha, Vernonanthura diffusa, Miconia cinnamomifolia, Piptocarpha macropoda and Luehea grandiflora. The Shannon diversity index $\left(H^{\prime}\right)$ was 3.31 nat.ind.$^{-1}$ and the Pielou evenness $\left(J^{\prime}\right)$ was 0.83 . In the mature forest, 623 individuals
\end{abstract}

\footnotetext{
${ }^{1}$ Recebido em 16.10.2006 e aceito para publicação em 29.03.2007.

${ }^{2}$ Pós-graduação em Ciência Florestal da Universidade Federal de Viçosa, 36570-000 Viçosa-MG, Brasil. E-mail:<sheilaicp@ yahoo.com.br>. ${ }^{3}$ DepartamentodeEngenhariaFlorestal da UniversidadeFederal de Viçosa. Bolsista ProdutividadeemPesquisa-CNPq.E-mail:<venancio@ufv.br>.

${ }^{4}$ Departamento de Engenharia Florestal da Universidade Federal do Espírito Santo, 29500-000 Alegre-ES.

${ }^{5}$ Departamento de Solos da Universidade Federal de Viçosa, Bolsista Produtividade em Pesquisa - CNPq

${ }^{6}$ Fundação Biodiversitas, Coordenadoria de Áreas Protegidas, 30430-130 Belo Horizonte-MG.
} 
were identified with distribution among 31 families and 78 species. Based on their importance value (IV), the most representative species were Euterpe edulis, Piptadenia gonoacantha, Nectandra lanceolata, Myrcia sphaerocarpa and Guapira opposita. The Shannon diversity index ( $\left.H^{\prime}\right)$ was 3.46 nat.ind..$^{-1}$ and the Pielou evenness ( $\left.J^{\prime}\right)$ was 0.79 . The diameter distributions of the four most abundant species in each forest site showed a distinct pattern, apparently related to the successional stage.

Keywords: Semideciduous forest, community structure and phytossociology.

\section{INTRODUÇÃO}

As florestas semidecíduas da Zona da Mata de Minas Gerais sofrem processos de perturbação semelhantes aos que são submetidos os demais ecossistemas brasileiros. Os poucos remanescentes florestais necessitam urgentemente de pesquisas básicas, no sentido de promover a conservação e a preservação dos fragmentos florestais da região (MARANGON et al., 2003).

A velocidade de devastação das florestas ameaça os ecossistemas florestais primários, confinando-os basicamente em áreas protegidas. Assim, os estudos direcionados aos estádios sucessionais das florestas secundárias são primordiais, uma vez que as florestas em regeneração constituem importante laboratório natural, capaz de gerar informações indispensáveis ao entendimento da dinâmica sucessional (RIBAS et al., 2003).

O conhecimento da organização estrutural das populações de espécies arbustivo-arbóreas, através de estudos fitossociológicos, é base para a definição de estratégias de manejo e conservação de remanescentes florestais e de restauração florestal em áreas degradadas. Informações sobre o número de indivíduos por espécie, espécies dominantes e espécies raras devem ser consideradas nos projetos de restauração florestal, visando à sustentabilidade dos ecossistemas a serem restaurados.

Este estudo teve por objetivo descrever a estrutura comunitária e a composição de espécies do componente arbustivo-arbóreo de dois estádios sucessionais de Floresta Estacional Semidecidual na Reserva Florestal Mata do Paraíso, em Viçosa, MG.

\section{MATERIAL E MÉTODOS}

O estudo foi conduzido na Reserva Florestal Mata do Paraíso $\left(20^{\circ} 45^{\prime} \mathrm{S}\right.$ e $\left.42^{\circ} 55^{\prime} \mathrm{W}\right)$, pertencente à Universidade Federal de Viçosa, que possui 195 ha e altitude variando de 690 a 800 m, no Município de Viçosa, MG (BRAZ et al., 2002).

R. Árvore, Viçosa-MG, v.31, n.5, p.823-833, 2007
Oclima na regiãoé do tipo Cwb(Köppen), mesotérmico com verões quentes e chuvosos e invernos frios e secos. A temperatura média anual é de $21,8^{\circ} \mathrm{C}$ e a precipitação pluviométrica média anual, de 1.314,2 mm (CASTRO et al., 1983). A vegetação da reserva é composta por trechos de Floresta Estacional Semidecidual (VELOSO et al., 1991), compondo um mosaico de diferentes estádios sucessionais e pequenas áreas de brejo (SILVA-JÚNIOR et al., 2004), formando um mosaico florestal, em função de diferentes épocas e graus de intervenção (LEAL-FILHO, 1992). Nos topos e encostas dos morros ocorre o Latossolo VermelhoAmarelo álico, enquanto nos terraços o solo é caracterizado como Podzólico Vermelho-Amarelo câmbico (MEIRANETO, 1997).

Os trechos escolhidos para este estudo situamse em solos e posições topográficas diferentes, apresentando diferentes históricos de perturbação e regeneração, sendo caracterizados por Silva-Júnior et al. (2004). Um desses trechos, denominado neste estudo floresta inicial, situa-se no terço inferior de uma encosta, em Latossolo Vermelho-Amarelo álico, e encontra-se em processo de regeneração florestal em pastagem de Melinis minutiflora $\mathrm{P}$. Beauv. desde 1963. O outro trecho de floresta, denominado floresta madura, constitui um núcleo de floresta bem preservado, situado em área de baixada, em solo Podzólico VermelhoAmarelo câmbico, estando livre de distúrbios antrópicos nas últimas quatro décadas.

Para o levantamento fitossociológico foram alocadas, de forma sistemática, 10 parcelas de 10 × 30 $\mathrm{m}$, com intervalos de $10 \mathrm{~m}$ entre parcelas, em cada estádio sucessional da floresta, totalizando uma área amostral de 0,6 ha. Todos os indivíduos arbustivo- arbóreos com diâmetro a $1,30 \mathrm{~m}$ do solo (DAP) $\geq 4,8 \mathrm{~cm}$ foram identificados e medidos. $\mathrm{O}$ material botânico foi identificado por meio de consultas ao Herbário VIC da Universidade Federal de Viçosa, MG, e as espécies foram classificadas em famílias de acordo com o sistema do Angiosperm Phylogeny Group II (APG II, 2003). 
Foram calculados os seguintes parâmetros fitossociológicos clássicos descritos por MuellerDombois e Ellenberg (1974): freqüência, dominância e densidade absolutas e relativas e valor de importância (VI). Também foram calculados o índice de diversidade de Shannon ( $\left.H^{\prime}\right)$ e a equabilidade de Pielou ( $\left.J^{\prime}\right)$ (BROWER e ZAR, 1984). Os cálculos foram efetuados pelo programa FITOPAC 1 (SHEPHERD, 1996). Os valores do índice de diversidade de Shannon dos dois trechos de floresta estudados foram comparados pelo teste t, de Hutcheson (ZAR, 1996).

Distribuições de densidades das árvores por classes de diâmetro foram plotadas empregando-se intervalos de classes regulares de $10,0 \mathrm{~cm}$ para todos os indivíduos amostrados e para as quatro espécies com maiores valores de importância em cada estádio sucessional da floresta.

Amostras dos solos das florestas inicial e madura (camada $0-10 \mathrm{~cm}$ ) foram submetidas às análises química e textural no Departamento de Solos da Universidade Federal de Viçosa. As variáveis edáficas obtidas nas florestas inicial e madura foram comparadas utilizandose o teste t, de Student.

\section{RESULTADOS E DISCUSSÃO}

Os solos das florestas inicial e madura se distinguiram quanto à classe textural, apresentando diferenças significativas em relação aos teores de areia e silte. $\mathrm{O}$ solo da floresta madura apresentou maiores teores de macronutrientes ( $\mathrm{P}, \mathrm{K}, \mathrm{Ca}$ e $\mathrm{Mg}$ ) e menores teores de $\mathrm{Al}$, resultando em valores superiores de soma de bases (SB) e saturação por bases (V), além do $\mathrm{pH}$ mais elevado e do maior teor de matéria orgânica (MO) (Quadro 1).

Essas diferenças edáficas corroboram o padrão de distribuição dos solos na região de Viçosa, MG, em que nas porções mais altas e inclinadas os solos são distróficos e com altos teores de alumínio trocável, enquanto nas baixadas e nos terrenos planos os solos apresentam maior fertilidade (CÔRREA, 1983).

As características edáficas distintas apresentadas pelos solos das florestas inicial e madura são importantes, pois mostram a influência da qualidade do solo no processo de sucessão, podendo acelerar esse processo nos ambientes mais férteis.

Ao todo foram amostrados 1.022 indivíduos com diâmetro do tronco a $1,30 \mathrm{~m}$ do solo (DAP) $\geq 4,8 \mathrm{~cm}$, totalizando uma área amostral de 0,3 ha em cada um
Quadro 1 - Variáveis químicas e texturais de 20 amostras do solo superficial (camada de $0-10 \mathrm{~cm}$ ) coletadas nos estádios floresta inicial e floresta madura na Reserva Florestal Mata do Paraíso, em Viçosa, MG

Table 1 -Chemical and textural variables of topsoil samples $(0-10 \mathrm{~cm}$ deep $)$ collected in the initial and mature successional stages in the Forest Reserve Mata do Paraíso in Viçosa, MG

\begin{tabular}{|c|c|c|c|}
\hline & $\frac{\text { Floresta Inicial }}{\text { Média } \pm \text { Desvio }}$ & $\frac{\text { Floresta Madura }}{\text { Média } \pm \text { Desvio }}$ & $\mathrm{P}^{1}$ \\
\hline pH em $\mathrm{H}_{2} \mathrm{O}$ & $4,47 \pm 0,09$ & $5,38 \pm 0,56$ & $0,000 * *$ \\
\hline & $43 \pm 0,45$ & $2,55 \pm 0,94$ & $0,003 * *$ \\
\hline $\mathrm{K}\left(\mathrm{mg} \cdot \mathrm{dm}^{-3}\right)$ & $, 70 \pm 3,02$ & $66,70 \pm 19$ & $0,000 * *$ \\
\hline $\mathrm{Ca}\left(\mathrm{cmol}_{\mathrm{c}} \cdot \mathrm{dm}^{-3}\right)$ & $0,814 \pm 0$ & $5,27 \pm 1,78$ & $0,000 * *$ \\
\hline & & $45+0$ & $0,000 * *$ \\
\hline & & & $0,000 * *$ \\
\hline $\left.\mathrm{ol} \cdot \mathrm{dm}^{-3}\right)$ & ) $9,11=$ & & $0,000 * *$ \\
\hline & & & $0,000 * *$ \\
\hline & & & $0,000 * *$ \\
\hline & & & $0,003 * *$ \\
\hline & & 57,66 & $0,000 * *$ \\
\hline & $47,13 \pm 12,40$ & & $0,000 * *$ \\
\hline $\mathrm{MO}\left(\text { dag. } \cdot \mathrm{kg}^{-1}\right)^{2}$ & & & $0,001 * *$ \\
\hline Areia (\%) & $38,70 \pm 3,23$ & $23,50 \pm 13,77$ & $0,003 * *$ \\
\hline Argila (\%) & $50,30 \pm 5,12$ & $55,10 \pm 11,32$ & $0,237^{\mathrm{NS}}$ \\
\hline Silte (\%) & $11,00 \pm 5,03$ & $21,40 \pm 3,69$ & $0,000 * *$ \\
\hline
\end{tabular}

${ }^{1}$ As comparações foram efetuadas pelo teste t de Student $(n=10)$, em que: ** significância a $1 \%$ de probabilidade; ${ }^{\text {NS }}$ : ausência de significância; ${ }^{2}$ SB: soma de bases trocáveis; $t$ : capacidade de troca catiônica efetiva; $\mathrm{T}$ : capacidade de troca catiônica a pH 7,0; V: índice de saturação por bases; m: índice de saturação por alumínio; e MO: matéria orgânica.

dos dois estádios sucessionais estudados. Apenas 33 espécies foram comuns às duas áreas de estudo. Na floresta inicial foram amostrados 399 indivíduos, totalizando $18,4 \mathrm{~m}^{2} \cdot \mathrm{ha}^{-1}$ de área basal e densidade de 1.330 indivíduos por hectare (Quadro 2). Na floresta madura foram identificados 623 indivíduos, alcançando $38,2 \mathrm{~m}^{2}$.ha- ${ }^{-1}$ de área basal e 2.077 indivíduos por hectare (Quadro 3).

$\mathrm{Na}$ floresta inicial, as espécies que apresentaram os maiores valores de densidade relativa foram Piptadenia gonoacantha $(\mathrm{DR}=12,0 \%)$, Vernonanthura diffusa $(\mathrm{DR}=11,0 \%)$, Piptocarpha macropoda $(\mathrm{DR}=7,5 \%)$, Miconia cinnamomifolia (DR $=6,8 \%$ ) e Psychotria sessilis ( $\mathrm{DR}=5,5 \%)$. Na floresta madura, as espécies que se destacaram em densidade relativa foram Euterpe edulis $(\mathrm{DR}=19,9 \%)$, Myrcia sphaerocarpa $(\mathrm{DR}=7,4 \%)$, Guapira opposita (DR =7,1\%), Guarea macrophylla $(\mathrm{DR}=4,7 \%)$ e Nectandra lanceolata $(\mathrm{DR}=4,7 \%)$ (Quadros 2 e 3).

R. Árvore, Viçosa-MG, v.31, n.5, p.823-833, 2007 
Quadro 2 - Espécies arbustivo-arbóreas amostradas no estádio inicial de sucessão, na Reserva Mata do Paraíso, em Viçosa, MG: $\mathrm{N}$. ind. = número de indivíduos nas parcelas, $\mathrm{P}=$ número de parcelas em que ocorrem, $\mathrm{AB}$ = área basal e VI = valor de importância

Table 2 - Shrub and tree species recorded in initial successional stage, in the Mata do Paraíso Reserve, in Viçosa, MG: $N$. ind.$=$ number of individuals in the plots; $P=$ number of plots with the species, $A B=$ basal area; $V I=$ important value

\begin{tabular}{|c|c|c|c|c|c|c|c|c|}
\hline \multirow[t]{3}{*}{ Espécies } & \multirow{3}{*}{$\begin{array}{l}\text { N. } \\
\text { ind. }\end{array}$} & \multicolumn{2}{|c|}{ Densidade } & \multicolumn{2}{|c|}{ Freqüência } & \multicolumn{2}{|c|}{ Dominância } & \multirow[t]{2}{*}{ VI } \\
\hline & & Abs & Rel. & Abs. & Rel. & Abs. & Rel. & \\
\hline & & ind.ha $\mathrm{a}^{-1}$ & $\%$ & \multicolumn{2}{|c|}{$\%$} & $\mathrm{~m}^{2} \cdot \mathrm{ha}^{-1}$ & $\%$ & $\%$ \\
\hline Piptadenia gonoacantha (Mart.)J.F.Macbr. & 48 & 160,0 & 12,03 & 100,0 & 5,56 & 5,4638 & 29,7 & 47,2 \\
\hline Vernonanthura diffusa (Less.) H.Robinson & 44 & 146,7 & 11,03 & 80,0 & 4,44 & 1,3730 & 7,46 & 22,9 \\
\hline Piptocarpha macropoda (DC.) Baker & 30 & 100,0 & 7,52 & 70,0 & 3,89 & 1,7494 & 9,51 & 20,9 \\
\hline Miconia cinnamomifolia (DC.) Naudin & 27 & 90,0 & 6,77 & 90,0 & 5,00 & 2,0484 & 11,1 & 22,9 \\
\hline Psychotria sessilis Vell. & 22 & 73,3 & 5,51 & 70,0 & 3,89 & 0,1822 & 0,99 & 10,3 \\
\hline Siparuna guianensis Aubl. & 21 & 70,0 & 5,26 & 90,0 & 5,00 & 0,2211 & 1,20 & 11,4 \\
\hline Sparattosperma leucanthum (Vell.) K. Schum. & 17 & 56,7 & 4,26 & 70,0 & 3,89 & 0,5625 & 3,06 & 11,2 \\
\hline Senna macranthera (Vell.) Irwin \& Barneby & 17 & 56,7 & 4,26 & 80,0 & 4,44 & 0,5683 & 3,09 & 11,7 \\
\hline Annona cacans Warm. & 14 & 46,7 & 3,51 & 60,0 & 3,33 & 0,9607 & 5,22 & 12,06 \\
\hline Nectandra oppositifolia Nees & 13 & 43,3 & 3,26 & 70,0 & 3,89 & 0,3157 & 1,72 & 8,86 \\
\hline Trichilia pallida $\mathrm{Sw}$ & 13 & 43,3 & 3,26 & 50,0 & 2,78 & 0,1234 & 0,67 & 6,71 \\
\hline Luehea grandiflora Mart. & 11 & 36,7 & 2,76 & 50,0 & 2,78 & 1,6861 & 9,17 & 14,7 \\
\hline Xylopia sericea A St.-Hil. & 10 & 33,3 & 2,51 & 60,0 & 3,33 & 0,2217 & 1,21 & 7,05 \\
\hline Erythroxylum pelleterianum A St.-Hil. & 10 & 33,3 & 2,51 & 50,0 & 2,78 & 0,1081 & 0,59 & 5,87 \\
\hline Solanum leucodendron Sendtn. & 8 & 26,7 & 2,01 & 50,0 & 2,78 & 0,3229 & 1,76 & 6,45 \\
\hline Zanthoxylum rhoifolium Lam. & 7 & 23,3 & 1,75 & 40,0 & 2,22 & 0,1513 & 0,82 & 4,80 \\
\hline Vismia guianensis (Aubl.) Pers. & 6 & 20 & 1,50 & 50,0 & 2,78 & 0,0864 & 0,47 & 4,75 \\
\hline Anadenanthera macrocarpa (Benth.) Brenan & 6 & 20,0 & 1,50 & 20,0 & 1,11 & 0,1467 & 0,80 & 3,41 \\
\hline Cecropia glaziovi Snethlage & 6 & 20,0 & 1,50 & 40,0 & 2,22 & 0,1333 & 0,72 & 4,45 \\
\hline Stryphnodendron polyphyllum Mart. & 5 & 16,7 & 1,25 & 30,0 & 1,67 & 0,1057 & 0,57 & 3,49 \\
\hline Myrcia fallax (Rich.) DC. & 5 & 16,7 & 1,25 & 50,0 & 2,78 & 0,1417 & 0,77 & 4,80 \\
\hline Protium warmingiana March, L. & 4 & 13,3 & 1,00 & 30,0 & 1,67 & 0,0477 & 0,26 & 2,93 \\
\hline Senna multijuga (Rich.) H. S. Irwin \& Barneby & 4 & 13,3 & 1,00 & 30,0 & 1,67 & 0,3012 & 1,64 & 4,31 \\
\hline Lacistema pubescens Mart. & 4 & 13,3 & 1,00 & 30,0 & 1,67 & 0,0484 & 0,26 & 2,93 \\
\hline Nectandra lanceolata Nees & 3 & 10,0 & 0,75 & 30,0 & 1,67 & 0,0612 & 0,33 & 2,75 \\
\hline Myrcia rostrata DC. & 3 & 10,0 & 0,75 & 30,0 & 1,67 & 0,0351 & 0,19 & 2,61 \\
\hline Matayba elaeagnoides Radlk. & 3 & 10,0 & 0,75 & 30,0 & 1,67 & 0,0283 & 0,15 & 2,57 \\
\hline Cecropia hololeuca Miq. & 3 & 10,0 & 0,75 & 30,0 & 1,67 & 0,4547 & 2,47 & 4,89 \\
\hline Schefflera morototoni (Aubl.) Maguire & 2 & 6,7 & 0,50 & 20,0 & 1,11 & 0,0255 & 0,14 & 1,75 \\
\hline Syagrus romanzoffiana (Cham.) Glassman & 2 & 6,7 & 0,50 & 10,0 & 0,56 & 0,0764 & 0,42 & 1,47 \\
\hline Sapium glandulatum (Vel1.) Pax & 2 & 6,7 & 0,50 & 20,0 & 1,11 & 0,0541 & 0,29 & 1,91 \\
\hline Dalbergia nigra Allemão ex Benth. & 2 & 6,7 & 0,50 & 10,0 & 0,56 & 0,0370 & 0,20 & 1,26 \\
\hline Cabralea canjerana (Vell.) Mart. & 2 & 6,7 & 0,50 & 20,0 & 1,11 & 0,0395 & 0,21 & 1,83 \\
\hline Trichilia lepidota Mart. & 2 & 6,7 & 0,50 & 10,0 & 0,56 & 0,0158 & 0,09 & 1,14 \\
\hline Dictyoloma vandellianum A H. L. Juss & 2 & 6,7 & 0,50 & 20,0 & 1,11 & 0,0482 & 0,26 & 1,87 \\
\hline Casearia sylvestris $\mathrm{Sw}$. & 2 & 6,7 & 0,50 & 20,0 & 1,11 & 0,0647 & 0,35 & 1,96 \\
\hline Jacaranda macrantha Cham. & 1 & 3,3 & 0,25 & 10,0 & 0,56 & 0,0106 & 0,06 & 0,86 \\
\hline Protium heptaphyllum (Aubl.) Marchand & 1 & 3,3 & 0,25 & 10,0 & 0,56 & 0,0117 & 0,06 & 0,87 \\
\hline Alchornea glandulosa Poepp. & 1 & 3,3 & 0,25 & 10,0 & 0,56 & 0,0325 & 0,18 & 0,98 \\
\hline Croton floribundus Spreng. & 1 & 3,3 & 0,25 & 10,0 & 0,56 & 0,0072 & 0,04 & 0,85 \\
\hline Hyeronima alchorneoides Allemão & 1 & 3,3 & 0,25 & 10,0 & 0,56 & 0,0297 & 0,16 & 0,97 \\
\hline Manihot pilosa Pohl & 1 & 3,3 & 0,25 & 10,0 & 0,56 & 0,0106 & 0,06 & 0,86 \\
\hline Apuleia leiocarpa (Vogel) J. F. Macbr. & 1 & 3,3 & 0,25 & 10,0 & 0,56 & 0,0101 & 0,05 & 0,86 \\
\hline Bauhinia forficata Link & 1 & 3,3 & 0,25 & 10,0 & 0,56 & 0,0140 & 0,08 & 0,88 \\
\hline Machaerium nyctitans (Vells.) Benth. & 1 & 3,3 & 0,25 & 10,0 & 0,56 & 0,0081 & 0,04 & 0,85 \\
\hline
\end{tabular}


Quadro 2-Cont.

Table 2 - Cont.

\begin{tabular}{|c|c|c|c|c|c|c|c|c|}
\hline \multirow{3}{*}{ Espécies } & \multirow{3}{*}{$\begin{array}{c}\text { N. } \\
\text { ind. }\end{array}$} & \multicolumn{2}{|c|}{ Densidade } & \multicolumn{2}{|c|}{ Freqüência } & \multicolumn{2}{|c|}{ Dominância } & \multirow[t]{2}{*}{ VI } \\
\hline & & Abs & Rel. & Abs. & Rel. & Abs. & Rel. & \\
\hline & & ind.ha $^{-1}$ & $\%$ & \multicolumn{2}{|c|}{$\%$} & $\mathrm{~m}^{2} \cdot \mathrm{ha}^{-1}$ & $\%$ & $\%$ \\
\hline Peltophorum dubium (Spreng.) Taub. & 1 & 3,3 & 0,25 & 10,0 & 0,56 & 0,0153 & 0,08 & 0,89 \\
\hline Ocotea corymbosa (Meisn.) Mez, & 1 & 3,3 & 0,25 & 10,0 & 0,56 & 0,0756 & 0,41 & 1,22 \\
\hline Guatteria nigrescens Mart. & 1 & 3,3 & 0,25 & 10,0 & 0,56 & 0,0255 & 0,14 & 0,94 \\
\hline Sorocea bonplandii (Baill.) W. C. Burger & 1 & 3,3 & 0,25 & 10,0 & 0,56 & 0,0446 & 0,24 & 1,05 \\
\hline Guapira opposita (Vell.) Reitz & 1 & 3,3 & 0,25 & 10,0 & 0,56 & 0,0240 & 0,13 & 0,94 \\
\hline Colubrina glandulosa Perkins & 1 & 3,3 & 0,25 & 10,0 & 0,56 & 0,0223 & 0,12 & 0,93 \\
\hline Prunus sellowii Koehne & 1 & 3,3 & 0,25 & 10,0 & 0,56 & 0,0146 & 0,08 & 0,89 \\
\hline Carpotroche brasiliensis (Raddi) Endl. & 1 & 3,3 & 0,25 & 10,0 & 0,56 & 0,0106 & 0,06 & 0,86 \\
\hline Casearia gossypiosperma Briq. & 1 & 3,3 & 0,25 & 10,0 & 0,56 & 0,0117 & 0,06 & 0,87 \\
\hline Solanum cernnum Vell. & 1 & 3,3 & 0,25 & 10,0 & 0,56 & 0,0068 & 0,04 & 0,84 \\
\hline
\end{tabular}

As espécies da floresta inicial que apresentaram os maiores valores de dominância relativa foram Piptadenia gonoacantha $(\mathrm{DoR}=29,7 \%)$, Miconia cinnamomifolia $(\mathrm{DoR}=11,1 \%)$, Luehea grandiflora $(\mathrm{DoR}=9,2 \%)$, Vernonanthura diffusa $(\mathrm{DoR}=7,5 \%)$ e Annona cacans (DoR =5,2\%). Na floresta madura, as espécies com maiores valores de dominância relativa foram Piptadenia gonoacantha $(\mathrm{DoR}=$ $17,9 \%)$, Nectandra lanceolata (DoR $=9,1 \%)$, Euterpe edulis (DoR $=8,7 \%)$, Allophylus edulis $($ DoR $=5,4 \%)$ e Nectandra oppositifolia $($ DoR $=$ $5,3 \%$ ) (Quadros 2 e 3).

Quadro 3 - Espécies arbustivo-arbóreas amostradas na floresta madura, na Reserva Mata do Paraíso, em Viçosa, MG: N. ind. = número de indivíduos, $\mathrm{P}=$ número de parcelas em que ocorrem, $\mathrm{AB}$ = área basal e $\mathrm{VI}=$ valor de importância

Table 3 - Shrub and tree species recorded in mature successional stage, in the Mata do Paraíso Reserve, in Viçosa, MG. $N$. ind.$=$ number of individuals; $P=$ number of plots with the species; $A B=$ basal area; $V I=$ important value

\begin{tabular}{|c|c|c|c|c|c|c|c|c|}
\hline \multirow{4}{*}{$\begin{array}{l}\text { Espécies } \\
\text { Euterpe edulis Mart. }\end{array}$} & \multirow{4}{*}{$\begin{array}{l}\text { N. } \\
\text { ind. } \\
124\end{array}$} & \multicolumn{2}{|c|}{ Densidade } & \multicolumn{2}{|c|}{ Freqüência } & \multicolumn{2}{|c|}{ Dominância } & \multirow{3}{*}{$\begin{array}{l}\text { VI } \\
\%\end{array}$} \\
\hline & & Abs & Rel. & Abs. & Rel. & Abs. & Rel. & \\
\hline & & ind.ha $^{-1}$ & $\%$ & \multicolumn{2}{|c|}{$\%$} & $\mathrm{~m}^{2} \cdot \mathrm{ha}^{-1}$ & $\%$ & \\
\hline & & 413 & 19,9 & 100,0 & 3,89 & 3,3394 & 8,74 & 32,5 \\
\hline Piptadenia gonoacantha (Mart.)J.F.Macbr. & 18 & 60,0 & 2,89 & 60,0 & 2,33 & 6,8403 & 17,91 & 23,1 \\
\hline Nectandra lanceolata Nees & 29 & 96,7 & 4,65 & 80,0 & 3,11 & 3,4573 & 9,05 & 16,8 \\
\hline Myrcia sphaerocarpa DC. & 46 & 153 & 7,38 & 80,0 & 3,11 & 1,2032 & 3,15 & 13,7 \\
\hline Guapira opposita (Vell.) Reitz & 44 & 147 & 7,06 & 90,0 & 3,50 & 0,9436 & 2,47 & 13,0 \\
\hline Nectandra oppositifolia Nees & 21 & 70,0 & 3,37 & 80,0 & 3,11 & 2,0402 & 5,34 & 11,8 \\
\hline Allophylus edulis (A St.-Hil., Cambess. \& A Juss) & 15 & 50,0 & 2,41 & 60,0 & 2,33 & 2,0731 & 5,43 & 10,2 \\
\hline Bauhinia forficata Link & 17 & 56,7 & 2,73 & 100,0 & 3,89 & 1,2772 & 3,34 & 9,96 \\
\hline Guarea macrophylla Vahl & 29 & 96,7 & 4,65 & 80,0 & 3,11 & 0,5781 & 1,51 & 9,28 \\
\hline Prunus sellowii Koehne & 25 & 83,3 & 4,01 & 90,0 & 3,50 & 0,5521 & 1,45 & 8,96 \\
\hline Alchornea glandulosa Poepp. & 15 & 50,0 & 2,41 & 70,0 & 2,72 & 1,3134 & 3,44 & 8,57 \\
\hline Cabralea canjerana (Vell.) Mart. & 14 & 46,7 & 2,25 & 50,0 & 1,95 & 1,3039 & 3,41 & 7,61 \\
\hline Hyeronima alchorneoides Allemão & 7 & 23,3 & 1,12 & 70,0 & 2,72 & 1,1300 & 2,96 & 6,81 \\
\hline Citronella paniculata (Mart.) R.A Howard & 15 & 50,0 & 2,41 & 90,0 & 3,50 & 0,2889 & 0,76 & 6,67 \\
\hline Allophylus sericeus Radlk. & 7 & 23,3 & 1,12 & 60,0 & 2,33 & 0,7821 & 2,05 & 5,51 \\
\hline Croton floribundus Spreng. & 4 & 13,3 & 0,64 & 30,0 & 1,17 & 1,2164 & 3,18 & 4,99 \\
\hline Pseudobombax grandiflorum (Cav.) A Robyns & 2 & 6,7 & 0,32 & 20,0 & 0,78 & 1,3804 & 3,61 & 4,71 \\
\hline Ficus insipida Willd. & 3 & 10,0 & 0,48 & 20,0 & 0,78 & 1,2484 & 3,27 & 4,53 \\
\hline Sorocea bonplandii (Baillon.) W.C.Burger & 10 & 33,3 & 1,61 & 50,0 & 1,95 & 0,2722 & 0,71 & 4,26 \\
\hline
\end{tabular}

- - - - - - - - - - - - - - - - - - - - - - - - - - - -

Continued... 
Quadro 3 - Cont

Table 3 - Cont.

\begin{tabular}{|c|c|c|c|c|c|c|c|c|}
\hline \multirow[t]{3}{*}{ Espécies } & \multirow{3}{*}{$\begin{array}{l}\mathrm{N} . \\
\text { ind. }\end{array}$} & \multicolumn{2}{|c|}{ Densidade } & \multicolumn{2}{|c|}{ Freqüência } & \multicolumn{2}{|c|}{ Dominância } & \multirow[t]{2}{*}{ VI } \\
\hline & & Abs & Rel. & Abs. & Rel. & Abs. & Rel. & \\
\hline & & ind.ha $^{-1}$ & $\%$ & \multicolumn{2}{|c|}{$\%$} & $\mathrm{~m}^{2} \cdot \mathrm{ha}^{-1}$ & $\%$ & $\%$ \\
\hline Protium warmingiana March, L. & 11 & 36,7 & 1,77 & 50,0 & 1,95 & 0,1788 & 0,47 & 4,18 \\
\hline Casearia gossypiosperma Briq. & 7 & 23,3 & 1,12 & 50,0 & 1,95 & 0,3791 & 0,99 & 4,06 \\
\hline Tovomitopsis saldanhae Engl. & 11 & 36,7 & 1,77 & 40,0 & 1,56 & 0,2182 & 0,57 & 3,89 \\
\hline Lonchocarpus muehlbergianus Hassl. & 8 & 26,7 & 1,28 & 50,0 & 1,95 & 0,2272 & 0,59 & 3,82 \\
\hline Xylosma salzmannii (Clos) Eichler & 8 & 26,7 & 1,28 & 50,0 & 1,95 & 0,1743 & 0,46 & 3,69 \\
\hline Trichilia lepidota Mart. & 11 & 36,7 & 1,77 & 40,0 & 1,56 & 0,1291 & 0,34 & 3,66 \\
\hline Machaerium nyctitans (Vells.) Benth. & 7 & 23,3 & 1,12 & 50,0 & 1,95 & 0,1860 & 0,49 & 3,56 \\
\hline Cupania vernalis Cambess. & 7 & 23,3 & 1,12 & 50,0 & 1,95 & 0,1686 & 0,44 & 3,51 \\
\hline Picramnia regnelli Engl. & 6 & 20,0 & 0,96 & 50,0 & 1,95 & 0,0805 & 0,21 & 3,12 \\
\hline Chrysophyllum flexиosum Mart. & 5 & 16,7 & 0,80 & 40,0 & 1,56 & 0,2305 & 0,60 & 2,96 \\
\hline Rollinia sylvatica (A St.-Hil.) Martius & 4 & 13,3 & 0,64 & 30,0 & 1,17 & 0,3743 & 0,98 & 2,79 \\
\hline Virola oleifera (Schott) A C. Sm. & 5 & 16,7 & 0,80 & 40,0 & 1,56 & 0,1384 & 0,36 & 2,72 \\
\hline Annonaceae sp1. & 1 & 3,3 & 0,16 & 10,0 & 0,39 & 0,7205 & 1,89 & 2,44 \\
\hline Myrcia fallax (Rich.) DC. & 4 & 13,3 & 0,64 & 40,0 & 1,56 & 0,0852 & 0,22 & 2,42 \\
\hline Zanthoxylum rhoifolium Lam. & 2 & 6,7 & 0,32 & 20,0 & 0,78 & 0,5051 & 1,32 & 2,42 \\
\hline Sapium glandulatum (Vell.) Pax & 4 & 13,3 & 0,64 & 40,0 & 1,56 & 0,0773 & 0,20 & 2,40 \\
\hline Senna multijuga (Rich.) H. S. Irwin \& Barneby & 1 & 3,3 & 0,16 & 10,0 & 0,39 & 0,6833 & 1,79 & 2,34 \\
\hline Platycyamus regnellii Benth. & 2 & 6,7 & 0,32 & 20,0 & 0,78 & 0,4300 & 1,13 & 2,22 \\
\hline Anadenanthera macrocarpa (Benth.) Brenan & 4 & 13,3 & 0,64 & 30,0 & 1,17 & 0,1008 & 0,26 & 2,07 \\
\hline Ocotea odorifera (Vell.) Rohwer & 4 & 13,3 & 0,64 & 30,0 & 1,17 & 0,0839 & 0,22 & 2,03 \\
\hline Miconia pusilliflora Beurl. & 4 & 13,3 & 0,64 & 30,0 & 1,17 & 0,0822 & 0,22 & 2,02 \\
\hline Inga edulis Mart. & 3 & 10,0 & 0,48 & 20,0 & 0,78 & 0,2635 & 0,69 & 1,95 \\
\hline Matayba leocodictya Radlk. & 4 & 13,3 & 0,64 & 30,0 & 1,17 & 0,0524 & 0,14 & 1,95 \\
\hline Matayba elaeagnoides Radlk. & 4 & 13,3 & 0,64 & 30,0 & 1,17 & 0,0509 & 0,13 & 1,94 \\
\hline Vernonanthura diffusa (Less.) H.Robinson & 2 & 6,7 & 0,32 & 20,0 & 0,78 & 0,1335 & 0,35 & 1,45 \\
\hline Casearia arborea (Rich.) Urb. & 2 & 6,7 & 0,32 & 20,0 & 0,78 & 0,1352 & 0,35 & 1,45 \\
\hline Siparuna arianae V. Pereira & 3 & 10,0 & 0,48 & 20,0 & 0,78 & 0,0500 & 0,13 & 1,39 \\
\hline Inga marginata Willd. & 3 & 10,0 & 0,48 & 20,0 & 0,78 & 0,0462 & 0,12 & 1,38 \\
\hline Coffea arabica $\mathrm{L}$. & 3 & 10,0 & 0,48 & 20,0 & 0,78 & 0,0226 & 0,06 & 1,32 \\
\hline Eugenia speciosa Cambess. & 2 & 6,7 & 0,32 & 20,0 & 0,78 & 0,0457 & 0,12 & 1,22 \\
\hline Amaioua guianensis Aubl. & 2 & 6,7 & 0,32 & 20,0 & 0,78 & 0,0340 & 0,09 & 1,19 \\
\hline Dalbergia nigra All. ex Benth. & 3 & 10,0 & 0,48 & 10,0 & 0,39 & 0,1200 & 0,31 & 1,18 \\
\hline Myrciaria cauliflora (Mart.) O. Berg & 2 & 6,7 & 0,32 & 20,0 & 0,78 & 0,0203 & 0,05 & 1,15 \\
\hline Swartzia myrtifolia Sm. & 2 & 6,7 & 0,32 & 20,0 & 0,78 & 0,0165 & 0,04 & 1,14 \\
\hline Cariniana estrellensis (Raddi) Kuntze & 1 & 3,3 & 0,16 & 10,0 & 0,39 & 0,1375 & 0,36 & 0,91 \\
\hline Acanthinophyllum ilicifolium (Spreng.) Burger & 2 & 6,7 & 0,32 & 10,0 & 0,39 & 0,0484 & 0,13 & 0,84 \\
\hline Endlicheria paniculata (Spreng.) J. F. Macbr. & 2 & 6,7 & 0,32 & 10,0 & 0,39 & 0,0315 & 0,08 & 0,79 \\
\hline Guatteria sp. & 1 & 3,3 & 0,16 & 10,0 & 0,39 & 0,0537 & 0,14 & 0,69 \\
\hline Casearia sylvestris $\mathrm{Sw}$. & 1 & 3,3 & 0,16 & 10,0 & 0,39 & 0,0549 & 0,14 & 0,69 \\
\hline Guatteria nigrescens Mart. & 1 & 3,3 & 0,16 & 10,0 & 0,39 & 0,0479 & 0,13 & 0,68 \\
\hline Himatanthus phagedaenicus (Mart.) Woodson & 1 & 3,3 & 0,16 & 10,0 & 0,39 & 0,0468 & 0,12 & 0,67 \\
\hline Solanum sp. & 1 & 3,3 & 0,16 & 10,0 & 0,39 & 0,0402 & 0,11 & 0,65 \\
\hline Seguieria langsdorffii Moq. & 1 & 3,3 & 0,16 & 10,0 & 0,39 & 0,0344 & 0,09 & 0,64 \\
\hline Apuleia leiocarpa (Vog.) Macbr. & 1 & 3,3 & 0,16 & 10,0 & 0,39 & 0,0306 & 0,08 & 0,63 \\
\hline Cestrum sp. & 1 & 3,3 & 0,16 & 10,0 & 0,39 & 0,0284 & 0,07 & 0,62 \\
\hline Manihot pilosa Pohl & 1 & 3,3 & 0,16 & 10,0 & 0,39 & 0,0217 & 0,06 & 0,61 \\
\hline Lacistema pubescens Mart. & 1 & 3,3 & 0,16 & 10,0 & 0,39 & 0,0208 & 0,05 & 0,60 \\
\hline Protium heptaphyllum (Aubl.) Marchand & 1 & 3,3 & 0,16 & 10,0 & 0,39 & 0,0140 & 0,04 & 0,59 \\
\hline Ocotea corymbosa (Meisn.) Mez & 1 & 3,3 & 0,16 & 10,0 & 0,39 & 0,0145 & 0,04 & 0,59 \\
\hline
\end{tabular}

R. Árvore, Viçosa-MG, v.31, n.5, p.823-833, 2007 
Quadro 3-Cont.

Table 3 - Cont.

\begin{tabular}{|c|c|c|c|c|c|c|c|c|}
\hline \multirow[t]{3}{*}{$\begin{array}{l}\text { Espécies } \\
\end{array}$} & \multirow{3}{*}{$\begin{array}{l}\mathrm{N} . \\
\text { ind. }\end{array}$} & \multicolumn{2}{|c|}{ Densidade } & \multicolumn{2}{|c|}{ Freqüência } & \multicolumn{2}{|c|}{ Dominância } & \multirow[t]{2}{*}{ VI } \\
\hline & & Abs & Rel. & Abs. & Rel. & Abs. & Rel. & \\
\hline & & ind.ha $^{-1}$ & $\%$ & & & $\mathrm{~m}^{2} \cdot \mathrm{ha}^{-1}$ & $\%$ & $\%$ \\
\hline Jacaranda macrantha Cham. & 1 & 3,3 & 0,16 & 10,0 & 0,39 & 0,0101 & 0,03 & 0,58 \\
\hline Nectandra sp. & 1 & 3,3 & 0,16 & 10,0 & 0,39 & 0,0098 & 0,03 & 0,58 \\
\hline Myrcia sp. & 1 & 3,3 & 0,16 & 10,0 & 0,39 & 0,0122 & 0,03 & 0,58 \\
\hline Garcinia gardneriana (Planch. \& Triana) Zappi & 1 & 3,3 & 0,16 & 10,0 & 0,39 & 0,0081 & 0,02 & 0,57 \\
\hline Pseudopiptadenia contorta DC. & 1 & 3,3 & 0,16 & 10,0 & 0,39 & 0,0060 & 0,02 & 0,57 \\
\hline Trichilia catigua A Juss. & 1 & 3,3 & 0,16 & 10,0 & 0,39 & 0,0077 & 0,02 & 0,57 \\
\hline Myrcia rostrata DC. & 1 & 3,3 & 0,16 & 10,0 & 0,39 & 0,0086 & 0,02 & 0,57 \\
\hline Psychotria carthaginensis Jacq. & 1 & 3,3 & 0,16 & 10,0 & 0,39 & 0,0068 & 0,02 & 0,57 \\
\hline Carpotroche brasiliensis (Raddi) Endl. & 1 & 3,3 & 0,16 & 10,0 & 0,39 & 0,0071 & 0,02 & 0,57 \\
\hline Vochysiaceae sp. & 1 & 3,3 & 0,16 & 10,0 & 0,39 & 0,0064 & 0,02 & 0,57 \\
\hline
\end{tabular}

As principais espécies (VI) na floresta inicial foram Piptadenia gonoacantha, Vernonanthura diffusa, Miconia cinnamomifolia, Piptocarpha macropoda, Luehea grandiflora, Annona cacans, Senna macranthera, Siparuna guianensis, Sparattosperma leucanthum e Psychotria sessilis, compondo 62,1\% do valor de importância, enquanto na floresta madura as espécies com maior VI foram Euterpe edulis, Piptadenia gonoacantha, Nectandra lanceolata, Myrcia sphaerocarpa, Guapira opposita, Nectandra oppositifolia, Allophylus edulis, Bauhinia forficata, Guarea macrophylla e Prunus sellowii, representando 49,8\% do valor de importância (Quadros 2 e 3).

Dentre as 10 espécies mais importantes na floresta inicial, Piptadenia gonoacantha, Vernonanthura diffusa, Miconia cinnamomifolia, Piptocarpha macropoda, Luehea grandiflora, Annona cacans, Senna macranthera e Sparattosperma leucanthum são pioneiras e secundárias iniciais, típicas de estádios iniciais da sucessão (GANDOLFI et al., 1995; MARTINS et al., 2002, 2004), o que corresponde ao histórico de regeneração desse trecho de floresta. Na floresta madura, Euterpe edulis, a espécie mais importante, é tolerante à sombra e típica de estádios sucessionais avançados da Floresta Atlântica (AIDAR et al., 2001; MORENO et al., 2003).

As famílias com maior valor de importância (VI) na floresta inicial foram, em ordem decrescente, Fabaceae, Asteraceae, Melastomataceae, Annonaceae e Malvaceae, e na floresta madura Fabaceae, Arecaceae, Lauraceae, Euphorbiaceae e Sapindaceae. Trabalhos desenvolvidos na região de Viçosa indicam que nos levantamentos florísticos de Florestas Estacionais Semidecíduas as famílias Fabaceae, Euphorbiaceae, Lauraceae, Myrtaceae,
Rubiaceae e Annonaceae têm assumido grande importância (PAULA et al., 2002; RIBAS et al., 2003; MARANGON et al., 2003; SILVA et al., 2003; SILVA et al., 2004a). A presença da família Lauraceae entre as de maior valor de importância na floresta madura caracteriza o estado mais avançado da sucessão nesse trecho de floresta, uma vez que esta é considerada indicativa da passagem da floresta pioneira para um estádio sucessional mais avançado no domínio da Floresta Atlântica (TABARELLI et al., 1994).

Os índices de diversidade de Shannon e a equabilidade de Pielou foram $\mathrm{H}^{\prime}=3,31$ nat.ind. ${ }^{-1}$, $\mathrm{J}^{\text {' }}$ $=0,83$ e H' $=3,46$ nat.ind. $.^{-1}, J^{\prime}=0,79$ nas florestas inicial e madura, respectivamente, sendo registrada diferença significativa pelo teste $\mathrm{t}$, de Hutcheson $(\mathrm{t}=1,93$; $\mathrm{p}$ $<0,05)$, na diversidade das florestas, indicando um padrão médio de diversidade e uma dominância ecológica baixa, ou seja, há alta heterogeneidade florística do componente arbustivo-arbóreo. Esses valores de diversidade são compatíveis com os valores obtidos em 10 trechos de floresta estudados na Reserva Florestal Mata do Paraíso em diferentes condições de relevo e sucessão, que variaram de 2,43 a 3,65 nat.ind. ${ }^{-1}$ (SILVA et al., 2004b). No entanto, a comparação entre índices de diversidade demanda cautela, uma vez que vários fatores relacionados à sucessão, aos critérios de inclusão e ao método de amostragem adotado podem interferir nos valores (SILVA et al., 2000).

A distribuição dos indivíduos arbustivo-arbóreos das florestas inicial e madura nas classes diamétricas apresentou um padrão de J-invertido, ou seja, alta concentração de indivíduos nas classes de menor diâmetro e redução acentuada no sentido das classes 
maiores (Figura 1). A classe de diâmetro entre 4,8 e $15 \mathrm{~cm}$ compreendeu $82,2 \%$ dos indivíduos da floresta inicial e 79,6\% dos indivíduos da floresta madura. A classe de 15 a $25 \mathrm{~cm}$ compreendeu $10,7 \%$ e 12,2\%; a classe diamétrica de $25 \mathrm{~cm}$ a $35 \mathrm{~cm}, 4,8 \%$ e $4,6 \%$; e a classe diamétrica de 35 a $45 \mathrm{~cm}, 2,3 \%$ e $1,4 \%$ dos indivíduos amostrados nas florestas inicial e madura, respectivamente. As classes diamétricas subseqüentes não apresentaram representantes na floresta inicial e baixas porcentagens pela floresta madura: $1,3 \%$ na classe de 45 a $55 \mathrm{~cm}, 0,5 \%$ na classe de 55 a $65 \mathrm{~cm}$ e $0,4 \%$ na classe superior a $65 \mathrm{~cm}$.

Com poucas exceções, considerando a comunidade como um todo, as curvas de distribuição diamétrica são unimodais, com as classes de menores diâmetros possuindo maior número de indivíduos (MEIRA-NETO e MARTINS, 2003). No entanto, a análise das espécies isoladamente revela que somente parte dessas espécies segue o mesmo padrão da comunidade (OLIVEIRAFILHO et al., 1994).

A distribuição diamétrica das quatro espécies da floresta inicial com maior VI apresentou o modelo exponencial negativo, caracterizado pela relação inversa entre a densidade e a área basal. Nesse grupo de espécies pertencentes aos estádios iniciais de sucessão, Vernonanthura diffusa e Piptocarpha macropoda (GANDOLFI et al., 1995; MARTINS et al., 2002, 2004) apresentam ciclo de vida curto, portanto não conseguem alcançar as classes diamétricas superiores (Figura 2). Piptadenia gonoacantha, também típica de estádios iniciais da sucessão (GANDOLFI et al., 1995; MARTINS et al., 2002, 2004), pode atingir um porte razoável, mas apresenta apenas indivíduos de menor diâmetro na floresta inicial, devido ao curto tempo de regeneração dessa floresta.

A distribuição diamétrica das quatro espécies do estádio sucessional maduro da floresta estudada com maior VI apresentou três padrões distintos (Figura 3). Euterpe edulis e Myrcia sphaerocarpa exibiram grande concentração de indivíduos nas duas primeiras classes diamétricas e ausência de representantes nas classes superiores. Essas espécies são consideradas umbrófilas (secundárias tardias) e não atingem grandes diâmetros, particularmente E. edulis, uma palmeira de caule delgado. Nectandra lanceolata apresentou tendência de redução decrescente no número de indivíduos em direção às classes de maior diâmetro. Piptadenia gonoacantha, espécie de início de sucessão, apresentou poucos representantes nas menores classes diamétricas e aumento na densidade das classes subseqüentes. Esse padrão indica que a espécie está enfrentando dificuldades em regenerar na floresta madura, tendendo a sair do sistema à medida que a sucessão avança.

Werneck et al. (2000) constataram grande redução na densidade de pioneiras durante estudo da dinâmica da comunidade de uma Floresta Decídua, principalmente nas classes inferiores de tamanho. Ressaltaram que as prováveis causas estariam associadas às alterações microambientais provocadas pelo crescimento das espécies do dossel e, ou, competição com as árvores pertencentes a grupos ecológicos mais tardios em uma escala sucessional. Assim, pode-se observar que, dentro de cada microrregião climática, outros fatores como altitude, química do solo, topografia, luminosidade etc., podem desempenhar papel importante na determinação da composição florística e na estrutura fitossociológica da comunidade (LIBERMAN et al., 1985).

Pode-se concluir que os dois trechos de floresta apresentam nítidas diferenças estruturais, que devem ser minimizadas com o tempo, à medida que a sucessão avança e o dossel da floresta inicial passa a fornecer maior sombreamento para o recrutamento de espécies tardias.

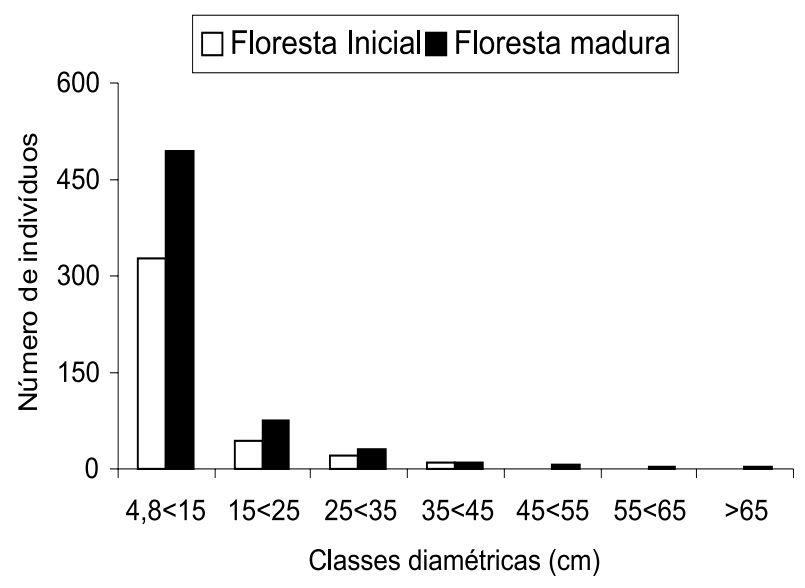

Figura 1 - Distribuição de diâmetro de indivíduos arbustivoarbóreos inventariados nos estádios sucessionais floresta inicial e floresta madura na Reserva Florestal Mata do Parais. Viçosa, MG, Brasil.

Figure 1 - Shrub and tree individuals diameter distribution in initial and mature successional stages in the Mata do Paraíso Forest Reserve. Viçosa, MG Brazil. 

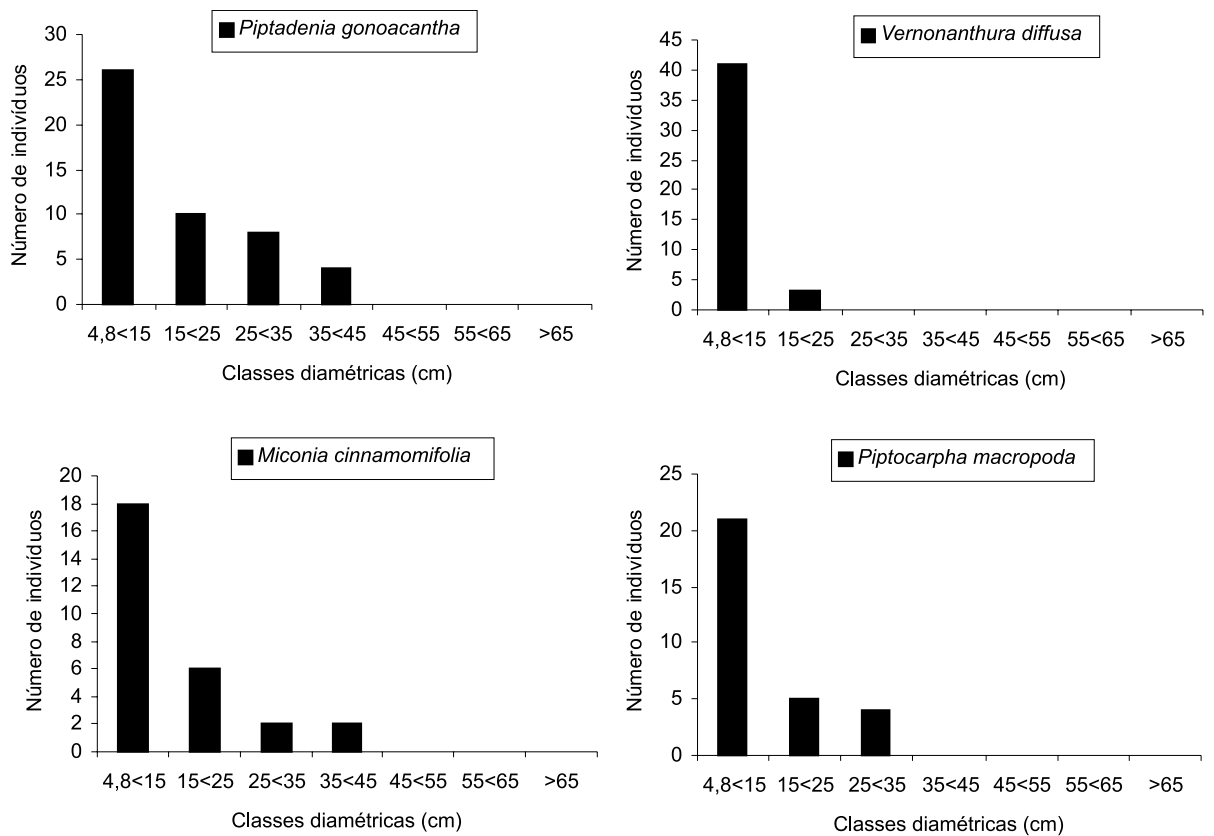

Figura 2 - Distribuição de diâmetro de indivíduos arbustivo-arbóreos das quatro espécies inventariadas com maior VI no estádio floresta inicial na Reserva Florestal Mata do Paraíso em Viçosa, MG, Brasil.

Figure 2-Shrub and tree individuals diameter distribution of four species surveyed with greater VI in initial successional stage in the Mata do Paraíso Forest Reserve in Viçosa, MG, Brazil.
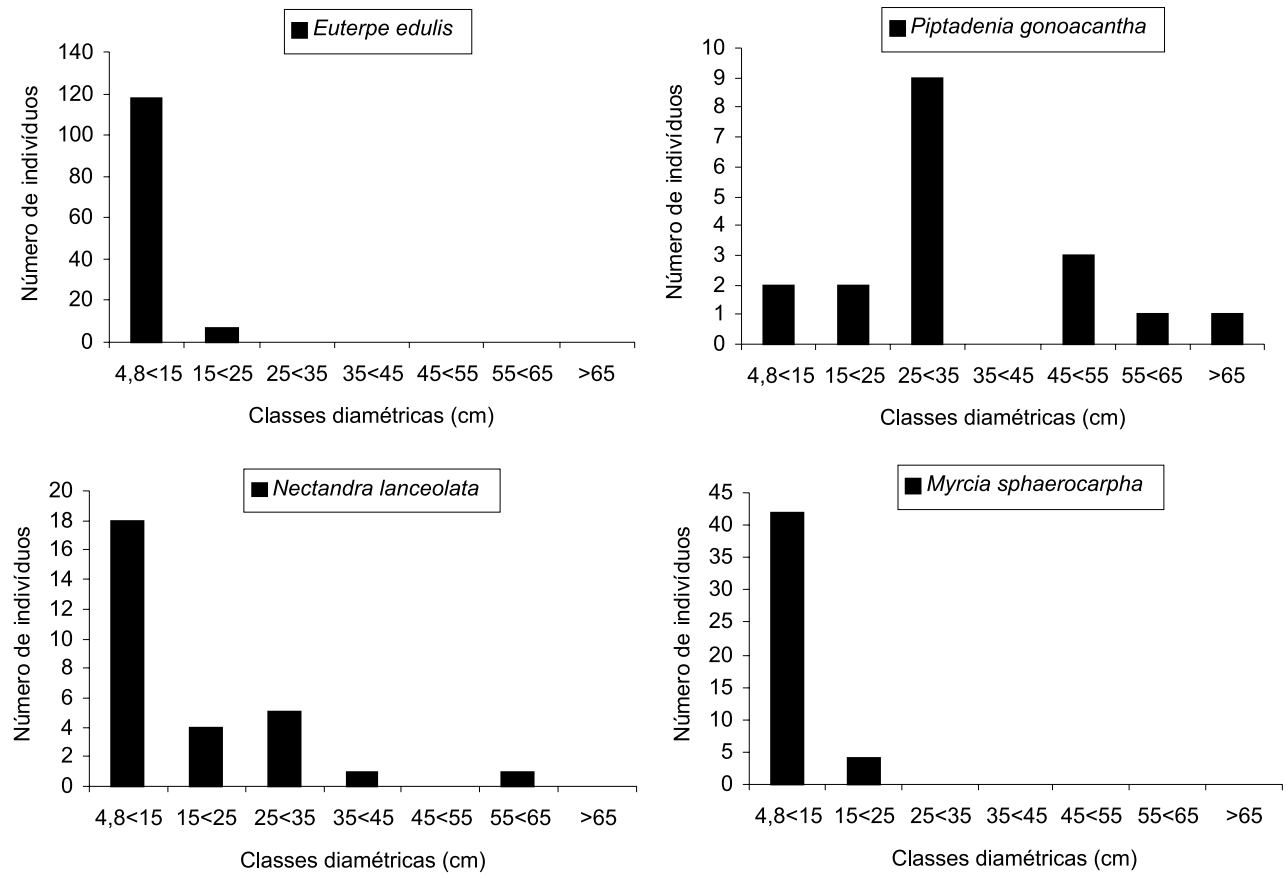

Figura 3 - Distribuição de diâmetro de indivíduos arbustivo-arbóreos das quatro espécies inventariadas com maior VI no estádio floresta madura na Reserva Florestal Mata do Paraíso, em Viçosa, MG, Brasil.

Figure 3 - Shrub and tree individuals diameter distribution of four species surveyed with greater VI in mature succecional stage in the Mata do Paraíso Forest Reserve in Viçosa, MG, Brazil. 


\section{REFERÊNCIAS}

AIDAR, M. P. M. et al.. Atlantic Forest succession over calcareous soil, Parque Estadual Turístico do Alto da Ribeira - PETAR, SP. Revista Brasileira de Botânica, v. 24, p.455-469, 2001.

ANGIOSPERM PHYLOGENY GROUP II. An update of the Angiosperm Phylogeny Group classification for the orders and families of flowering plants:

APGII. Botanical Journal of the

Linnaean Society, v.141, n.4, p.399-436, 2003.

BRAZ, D. M.; CARVALHO-OKANO, R. M.; KAMEYAMA, C. Acanthaceae da Reserva Florestal Mata do Paraíso, Viçosa, Minas Gerais. Revista Brasileira de Botânica, v.25, p.495-504, 2002.

BROWER, J. E.; ZAR, J.H. Field and laboratory methods for general ecology. Dubuque: W. M. C. Brown, 1984. 226p.

CASTRO, P. S.et al. Interceptação da chuva por mata natural secundária na região de Viçosa, MG. Revista Árvore, v.7, p.76-89, 1983.

CORRÊA, G. F. Modelo de evolução e mineralogia da fração argila de solos do Planalto de Viçosa. 1983. 87f.

Dissertação (Mestrado em Solos e Nutrição de Plantas). Universidade Federal de Viçosa, Viçosa, MG, 1983.

GANDOLFI, S.; LEITÃO-FILHO, H. F.; BEZERRA, C. L. F. Levantamento florístico e caráter sucessional das espécies arbustivo-arbóreas de uma Floresta Mesófila Semidecídua no município de Guarulhos, SP. Revista Brasileira de Biologia, v.55, n.4, p.753-767, 1995.

LEAL-FILHO, N. Caracterização do banco de sementes de três estádios de uma sucessão vegetal na Zona da Mata de Minas Gerais. 1992. 196f.

Dissertação(Mestrado em Ciência Florestal) Universidade Federal de Viçosa, Viçosa, MG, 1992.

LIBERMAN, M. et al. Small-scale altitudinal variation in lowland west tropical forest vegetation. Journal Ecology, v.73, p.505-516, 1985.
MARANGON, L. C.; SOARES; J. J.; FELICIANO, A. L. P. Florística arbórea da Mata da Pedreira, município de Viçosa, Minas Gerais. Revista Árvore, v.27, n.2, p.207-215, 2003.

MARTINS, S. V.; COUTINHO, M. P.; MARANGON, L. C. Composição florística e estrutura de uma floresta secundária no município de Cruzeiro-SP. Revista Árvore, v.26, n.1, p.35-41, 2002.

MARTINS, S. V. et al. Colonization of gaps produced by death of bamboo clumps in a Semideciduous Mesophytic Forest in southeastern Brazil. Plant Ecology, v.172, p.121-131, 2004.

MEIRA-NETO, J. A. A. Estudos florísticos, estruturais e ambientais nos estratos arbóreos e herbáceo-arbustivo de uma Floresta Estacional Semidecidual em Viçosa, MG. 1997. 154f. Tese (Doutorado em Ciências Biológicas). Universidade Estadual de Campinas, Campinas, 1997.

MEIRA-NETO, J. A. A.; MARTINS, F. R. Estrutura do sub-bosque herbáceo-arbustivo da Mata da Silvicultura, uma Floresta Estacional Semidecidual do município de Viçosa-MG. Revista Árvore, v.27, n.4, p.459-471, 2003.

MORENO, M. R.; NASCIMENTO, M. T.; KURTZ, B. C. Estrutura e composição florística do estrato arbóreo em duas zonas altitudinais na Mata Atlântica de encosta da região do Imbé, RJ. Acta Botânica Brasílica, v.17, n.3, p.371-386, 2003.

MUELLER-DOMBOIS, D.; ELLENBERG, H. Aims and methods in vegetation ecology. New York: Wiley \& Sons, 1974. 547p.

OLIVEIRA-FILHO, A. T.; SCOLFORO, J. R. S.; MELLO, J. M. Composição florística e estrutura de um remanescente de Floresta Semidecidual Montana em Lavras, MG. Revista Brasileira de Botânica, v. 17, n.2, p.167-182, 1994.

PAULA, A. et al. Alterações florísticas ocorridas num período de quatorze anos na vegetação arbórea de uma Floresta Estacional Semidecidual em Viçosa-MG. Revista Árvore, v.26, n.6, p.743-749, 2002. 
RIBAS, R. F. et al. Composição florística de dois trechos em diferentes etapas serais de uma Floresta Estacional Semidecidual em Viçosa, Minas Gerais. Revista Árvore, v.27, n.6, p.821-830, 2003.

SHEPHERD, G. J. FITOPAC 1, Manual do usuário. Campinas: UNICAMP, 1996.

SILVA, A. F.; FONTES, N. R.; LEITÃO-FILHO, H. Composição florística e estrutura horizontal do estrato arbóreo de um trecho da Mata da Biologia da Universidade Federal de Viçosa - Zona da mata de Minas Gerais. Revista Árvore, v.24, n.4, p.397-405, 2000.

SILVA, A. F. et al. Composição florística e grupos ecológicos das espécies de um trecho de Floresta Semidecídua Submontana da Fazenda São Geraldo, Viçosa-MG. Revista Árvore, v.27, n.3, p.311-319, 2003.

SILVA, N. R. S. et al. Composição florística e estrutura de uma Floresta Estacional Semidecidual Montana em Viçosa, MG. Revista Árvore, v.28, n.3, p.397-405, 2004a.

SILVA, C. T. et al. Avaliação temporal da florística arbórea de uma floresta secundária no município de Viçosa, Minas Gerais. Revista Árvore, v.28, n.3, p.429-441, 2004b.
SILVA-JÚNIOR, W. M. et al. Regeneração natural de espécies arbustivo-arbóreas em dois trechos de Floresta Estacional Semidecidual, Viçosa, MG. Scientia Forestalis, v.66, p.169-179, 2004.

TABARELLI, M.; VILLANI, J.P.; MANTOVANI, W. Estudo comparativo da vegetação de dois trechos de floresta secundária no Núcleo Santa Virgínia, Parque Estadual da Serra do Mar, SP. Revista do Instituto Florestal, v.6, p.1-11, 1994.

VELOSO, H. P.; RANGEL FILHO, A. L. R.; LIMA, J. C. A. Classificação da vegetação brasileira, adaptada a um sistema universal. Rio de Janeiro: Instituto Brasileiro de Geografia e Estatística, 1991.

WERNECK, M. S.; FRANCESCHINELLI, E. V.; TAMEIRÃO-NETO, E. Mudanças na florística e estrutura de uma Floresta Decídua durante um período de quatro anos (1994-1998), na região do Triângulo Mineiro, MG. Revista Brasileira de Botânica, v.23, n.4, p. 401-413. 2000.

ZAR, J. H. Biostatistical analysis. New Jersey: Prentice-Hall, 1996. 